\title{
Hallazgo de un Tumor Odontogénico Adenomatoide en una paciente adolescente
}

\author{
Jeremias Roman, ${ }^{1}$ Sebastian Miguelez, ${ }^{2}$ Ariel Monteagudo, ${ }^{3}$ Dr. Christian Oscar Mosca. ${ }^{4}$
}

\begin{abstract}
Resumen: El tumor odontogénico adenomatoide (TOA), fue descripto en 1905 por Steensland. En el año 2005, la OMS lo clasificó como una neoplasia benigna constituida por epitelio odontogénico con estroma fibroso sin ectomesénquima odontogénico. El diagnóstico diferencial del TOA debe hacerse con el quiste dentígero, ameloblastoma uniquístico y tumor odontogénico epitelial calcificante. En el presente artículo se presenta el caso de una paciente femenina de 14 años que concurre al servicio de odontología del "H.I.G.A. Presidente Perón de Avellaneda", que al análisis intraoral, se detectó un abombamiento de la tabla ósea vestibular de la zona anterior de la hemi-mandibula derecha a nivel de caninos y premolares, indoloro, de consistencia sólida, bordes definidos, sin crepitación, no identificado por la paciente con ausencia de la pieza 44 y persistencia del 84. Se realizó ortopantomografia de rutina y TAC de macizo cráneo-facial, para la planificación del abordaje quirúrgico y evaluar relación con estructuras nobles. Se decide intervenirla bajo anestesia general para la extirpación quirúrgica de la lesión, obteniendo en el estudio anatomopatología como resultado Tumor Odontogénico Adenomatoide o TOA, por lo que se realizaron controles durante 10 meses, continuando en la actualidad con los mismos. El pronóstico del TOA es bueno, la tasa de recurrencia es de $0,2 \%$. En nuestro caso la decisión fue de una cirugía conservadora sin regeneración ósea debido a la edad de la paciente, y la cercanía del paquete vasculonervioso dentario inferior y su ramal terminal mentoniana, en donde los controles posquirúrgicos demostraron una buena evolución con recuperación de la sensibilidad y la no recidiva de la entidad tumoral.
\end{abstract}

Palabras clave: tumor adenomatoide, diente no erupcionado, tumores odontogénicos, neoplasias.

\section{Descoberta de um tumor odontogênico adenomatóide em um paciente adolescente}

Resumo: O tumor odontogênico adenomatóide (TOA) foi descrito em 1905 por Steensland. Em 2005, a OMS classificou-a como uma neoplasia benigna constituída por epitélio odontogênico com estroma fibroso sem ectomesênquima odontogênico. O diagnóstico diferencial da TOA deve ser feito com o cisto dentígero, ameloblastoma unicístico e tumor odontogênico epitelial calcário. Este artigo apresenta o caso de uma paciente de 14 anos, do sexo feminino, que frequenta o serviço odontológico do "HIGA Presidente Perón da Avellaneda", que na análise intraoral detectou abaulamento da mesa óssea vestibular na região anterior do Hemi-mandíbula direita ao nível dos caninos e pré-molares, indolor, de consistência sólida, bordas definidas, sem crepitação, não identificada pelo paciente com ausência de dente 44 e persistência do dente 84 . Ortopantomografia de rotina e maciço craniofacial foram realizados para planejamento da abordagem cirúrgica e avaliação da relação com estruturas nobres. Optou-se por intervir sob anestesia geral para a retirada cirúrgica da lesão, obtendo no estudo anatomopatológico como resultado Tumor Odontogênico Adenomatóide ou TOA, para os quais foram realizados controles por 10 meses, continuando com eles atualmente. O prognóstico para TOA é bom, a taxa de recorrência é de 0,2\%. Em nosso caso, optou-se por uma cirurgia conservadora sem regeneração óssea devido à idade do paciente, e à proximidade do feixe neurovascular dentário inferior e seu ramo mandibular terminal, onde os controles pós-operatórios apresentaram boa evolução com recuperação da sensibilidade e a não recorrência da entidade tumoral.

Palavras-chave: tumor adenomatóide, dente não erupcionado, tumores odontogênicos, neoplasias.

${ }^{1}$ Residente Tercer Año del Hospital Interzonal General de Agudos Pte. Perón

${ }^{2}$ Odontólogo del Hospital Interzonal General de Agudos Pte. Perón. Especialista en Prótesis Dento buco maxilar.

${ }^{3}$ Odontólogo del Hospital Interzonal General de Agudos Pte. Perón.

${ }^{4}$ Especialista en Cirugía y Traumatología BMF. Doctor en Salud Pública en Sistema y Servicios de Salud. Profesor Adjunto del Departamento de Microbiología e Inmunología, Universidad Kennedy. Coordinador Académico del Doctorado en Salud Pública UCES. Docente Asesor Colaborador del Servicio de Odontología del Hospital Interzonal General de Agudos Pte. Perón. 


\title{
Finding of an Adenomatoid Odontogenic Tumor in an adolescent patient
}

\begin{abstract}
The adenomatoid odontogenic tumor (AOT) was described in 1905 by Steensland. In 2005, the WHO classified it as a benign neoplasm consisting of odontogenic epithelium with fibrous stroma without odontogenic ectomesenchyme. The differential diagnosis of AOT should be made with the dentigerous cyst, unicystic ameloblastoma and calcifying epithelial odontogenic tumor. This article presents the case of a 14-year-old female patient who attends the dental service of the "HIGA President Perón to Avellaneda", who, upon intraoral analysis, detected a bulging of the vestibular bone table in the anterior area of the Right hemi-mandible at the level of the canines and premolars, painless, of solid consistency, defined edges, without crepitation, not identified by the patient with absence of tooth 44 and persistence of 84 . Routine orthopantomography and craniofacial massif were performed to plan the surgical approach and evaluate the relationship with noble structures. It was decided to intervene under general anesthesia for the surgical removal of the lesion, obtaining in the anatomopathology study as a result Adenomatoid Odontogenic Tumor or TOA, for which controls were carried out for 10 months, continuing with them at present. The prognosis for TOA is good, the recurrence rate is $0.2 \%$. In our case, the decision was for conservative surgery without bone regeneration due to the age of the patient, and the proximity of the inferior dental nerve bundle and its terminal chin branch, where the postoperative controls showed a good evolution with recovery of sensitivity and the non-recurrence of the tumor entity.
\end{abstract}

Keywords: adenomatoid tumor, non-erupted tooth, odontogenic tumors, Neoplasms.

\section{Introducción}

Los tumores odontogénicos son patologías benignas o malignas, propias de los maxilares, que pueden tener origen en el epitelio odontogénico, tejido ectomesenquimático o ambos, con gran variabilidad clínica e histológica. ${ }^{1,2}$

El tumor odontogénico adenomatoide, fue descrito en 1905 por Steensland, quien lo consideró una variable del ameloblastoma. Harbitz en 1915 lo llamó adamantoma quístico. ${ }^{3}$ En 1948 Stafne, lo definió como tumor adenomatoide ameloblástico - adenoameloblastoma, por presentar estructuras que se asimilaban a conductos - glándulas, clasificándolo como una variante del ameloblastoma. En 1969, Philipsen y Birn propusieron el nombre que lo define en la actualidad, "tumor odontogénico adenomatoide" (TOA), aceptado en 1971 por la Organización Mundial de la Salud (OMS). ${ }^{4}$ Este término es el más idóneo por cuanto que estas lesiones son benignas y a diferencia de los ameloblastomas tiene una tendencia a la recidiva muy baja, haciendo innecesario el realizar tratamientos quirúrgicos amplios o agresivos. ${ }^{5}$ En el año 2005, la OMS lo clasificó como una neoplasia benigna constituida por epitelio odontogénico con estroma fibroso sin ectomesénquima odontogénico. ${ }^{6}$ Según la última clasificación de tumores odontogénicos de la OMS en el año 2017, el TOA, es considerado "tumor odontogénico epitelial benigno".,78

El TOA, es una neoplasia benigna, poco frecuente, ${ }^{5}$ que representa del 2 al $7 \%$ de todos los tumores odontogénicos benignos. Se caracteriza por su curso asintomático, de crecimiento lento pero agresivo, lo que provoca expansión de las corticales óseas y desplazamiento de raíces de piezas dentarias continuas, sin causar rizálisis de las mismas y con baja tendencia a la recidiva. Se presenta durante la segunda y tercer década de vida, afectando principalmente al sexo femenino, siendo el maxilar superior ${ }^{9}$ en el sector anterior el sitio predilecto $^{10}$ y asociado a una 
pieza retenida, generalmente los caninos superiores, ${ }^{9}$ y en forma intraósea. ${ }^{11}$ Según Sethi, es conocido por varios autores como el tumor de los "dos tercios", teniendo en cuenta que "dos tercios" ocurren en el maxilar superior, "dos tercios" se ven en el sexo femenino, "dos tercios" se asocian con dientes retenidos y "dos tercios" de los diente son los caninos. ${ }^{12}$ Aparenta tener predilección por la población asiática y raza negra. ${ }^{1}$

Existen tres formas o subvariedades clínicas, todas ellas dotan de la misma histología: ${ }^{5}$ un tipo folicular, extrafolicular y un tipo periférico. ${ }^{13}$ El tipo folicular, de localización central, intraoseo, representando el $73 \%$ de los hallazgos y se observa como un área quística radiolúcida unilocular, bien definida, ${ }^{5}$ aunque puede presentar pequeños focos radiopacos distribuidos en toda su extensión, ${ }^{10}$ relacionada a un diente no erupcionado o impactado, simulando la imagen de un quiste dentígero; la forma extrafolicular $(24 \%)^{5}$ así mismo de localización central, intraoseo, sin relación con un órgano dental, mostrando una imagen con las mismas características que el anterior, por encima o superpuesta a las raíces de dientes permanentes erupcionados, que puede prestar confusión con quistes periapicales y otras lesiones quísticas y tumorales de los maxilares; ${ }^{5}$ y por último la forma periférica (3\%), siendo esta la más infrecuente, extraoseo, que aparece como un fibroma gingival o épulis, unido a la encía vestibular, pudiendo llevar a un diagnóstico preoperatorio erróneo. ${ }^{5}$

El diagnóstico diferencial del TOA debe hacerse con el quiste dentígero, ameloblastoma uniquístico y tumor odontogénico epitelial calcificante. ${ }^{1,14}$
Teniendo en cuenta su baja tasa de recidiva $(0,2 \%)$ y que es una lesión benigna, el tratamiento del TOA es conservador, centrándose en la enucleación de la lesión y la pieza asociada, previa evaluación ortodóncica de la misma para determinar la posibilidad eruptiva por tracción hacia la arcada dentaria, y curetaje de la cavidad residual. ${ }^{9}$

En la literatura, también se reportan casos excepcionales de lesiones tumorales de gran tamaño, o con riesgo de fractura, donde se indicó la resección en bloque de la mandíbula o región anterior del maxilar, y otras situaciones donde debido a la gran cavidad ósea residual se recomienda la regeneración guiada mediante el uso de hueso liofilizado y membranas. ${ }^{5,15}$

El objetivo del presente artículo es analizar y mostrar un caso clínico con su diagnóstico clínico e histopatológico y plan de tratamiento; adicionalmente se hace una revisión de literatura.

\section{Reporte de caso}

Para la presente investigación fundamentalmente se protegieron los derechos del paciente, primeramente bajo el consentimiento firmado por la madre del representado y la autorización en el área de docencia del Hospital Interzonal General de Agudos Gral. Perón, respetando los principios éticos basado en la Declaración de Helsinki.

Se presentó en el servicio de odontología del "Hospital Interzonal General de Agudos Presidente Perón de Avellaneda", una paciente de sexo femenino de 14 años de edad, acompañada por su madre solicitando un certificado bucodental requerido para el 


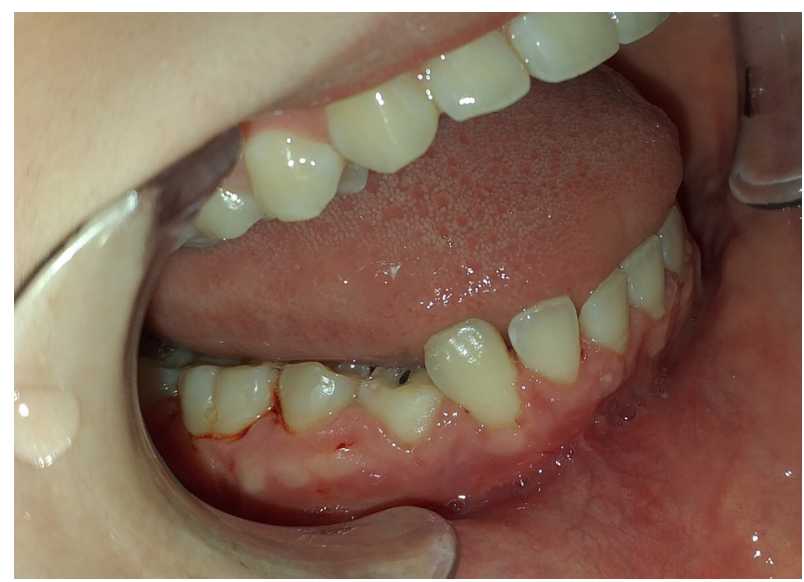

Figura 1. Examen clínico bucal. En la fotografía se puede observar la persistencia de la pieza 84 con ausencia de la pieza 44. Se puede visualizar que no hay cambios de la coloración de la encía o mucosa.

inicio escolar. Se realizó la historia clínica y anamnesis de la paciente.

Durante el examen cervicofacial no se observaron signos clínicos de relevancia a nivel extraoral. En el análisis intraoral, se detectó a la palpación un abombamiento de la tabla ósea vestibular de la zona anterior de la hemi-mandibula derecha a nivel de caninos y premolares, indoloro, de consistencia sólida, bordes definidos, sin crepitación, no identificado por la paciente; y en el examen dentario se observó buen estado general, permanencia de la pieza 84 y ausencia de la pieza dental 44 (Figura 1).

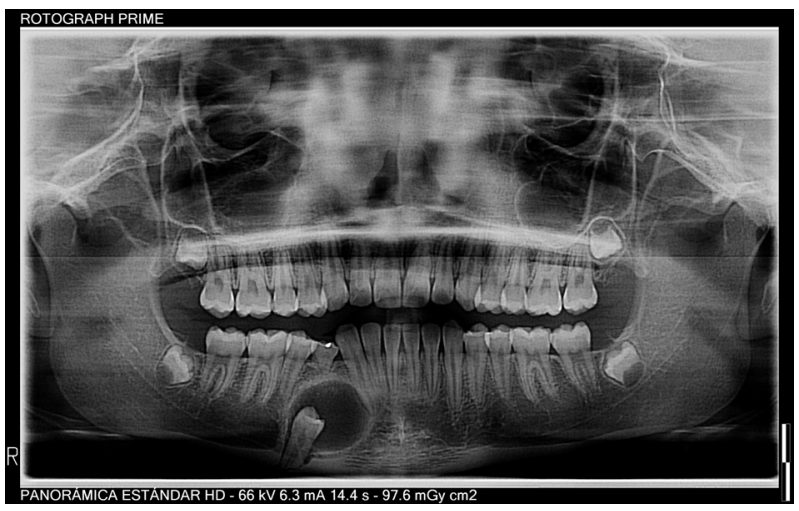

Figura 2. Ortopantomografía en la que se visualiza la retención de la pieza 44 rodeada la corona por una imagen radiolúcida compatible con una patología de características quísticas (siendo confirmado por la anatomía la presencia de un Tumor Odontogénico Adenomatoide). Se observa el desplazamiento de las raíces de la pieza 43 y 45, la íntima relación con el nervio dentario inferior y el orificio mentoniano

Se realizó ortopantomografia de rutina, y se observó en el hemi-maxilar inferior derecho, entre las piezas 43 y 45 una lesión radiolúcida unilocular, circunscripta por un borde óseo esclerótico bien definido, de $20 \mathrm{~mm}$ por $18 \mathrm{~mm}$, con su límite inferior próximo a la basal mandibular, desplazamiento radicular de las piezas contiguas, envolviendo la corona de la pieza 44 incluida (Figura 2). Se solicitó TAC de macizo cráneo-facial, para la planificación del abordaje quirúrgico y evaluar relación con estructuras nobles (Figura 3 A,B,C y D).
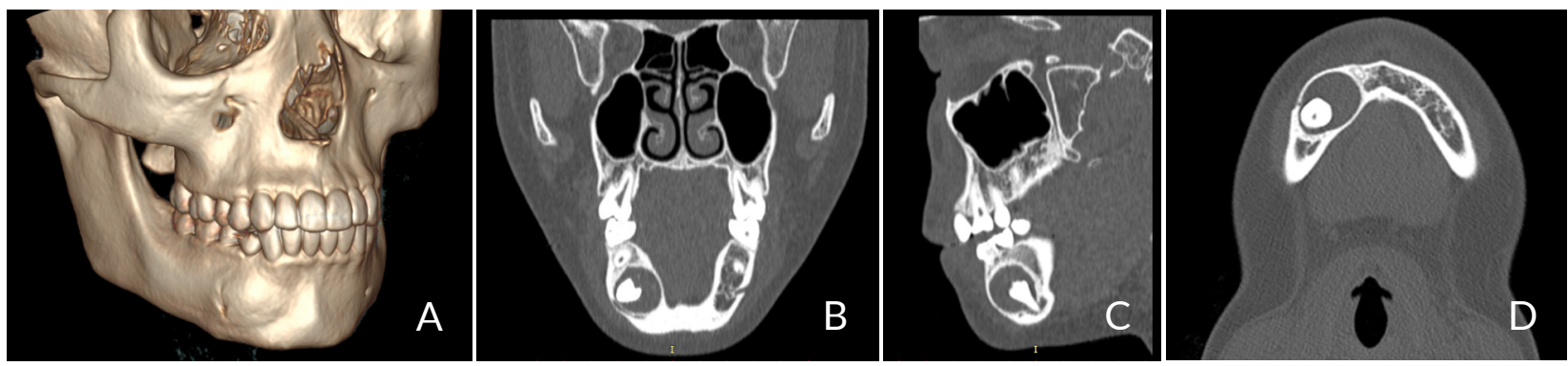

Figura 3. Tomografía Computada. En la imagen A de reconstrucción $3 d$ se visualiza el soplamiento de la tabla vestibular y la salida del conducto mentoniano. En la imagen B se observa el adelgazamiento de la tabla vestibular y lingual con el soplamiento que realiza la lesión en sentido V-L. En las imágenes $C$ y $D$ se observa el íntimo contacto con el conducto mentoniano. 
Realizado el diagnóstico de la lesión se solicitaron estudios pre-quirúrgicos para la intervención bajo anestesia general, con el objetivo de realizar la enucleación completa de la entidad y la pieza dentaria 44 asociada.

Se ingresó la paciente a quirófano, y se realizó anestesia general, intubación nasotraqueal, antisepsia de la región facial con yodopovidona $10 \%$, colocación de tapón faríngeo y campos estériles, e infiltración con lidocaína/epinefrina 0,2\%.

Se colocaron arcos peine tipo convencional en ambos maxilares y se procedió a realizar bloqueo intermaxilar intraquirúrgico, con el objeto de estabilizar la mandíbula y conservar la oclusión (Figura 4).

Luego se abordó bajo una incisión paramarginal horizontal de espesor total desde la pieza 41 a 45, con bisturí frío tipo bad-parker $n^{\circ} 3$ hoja $n^{\circ} 15$ y electrobisturí, para generar hemostasia, legrado de colgajo muco-perióstico, localización y reparo de nervio mentoniano, el cual se encontraba bifurcado(Figura 5), ostectomía con martillo

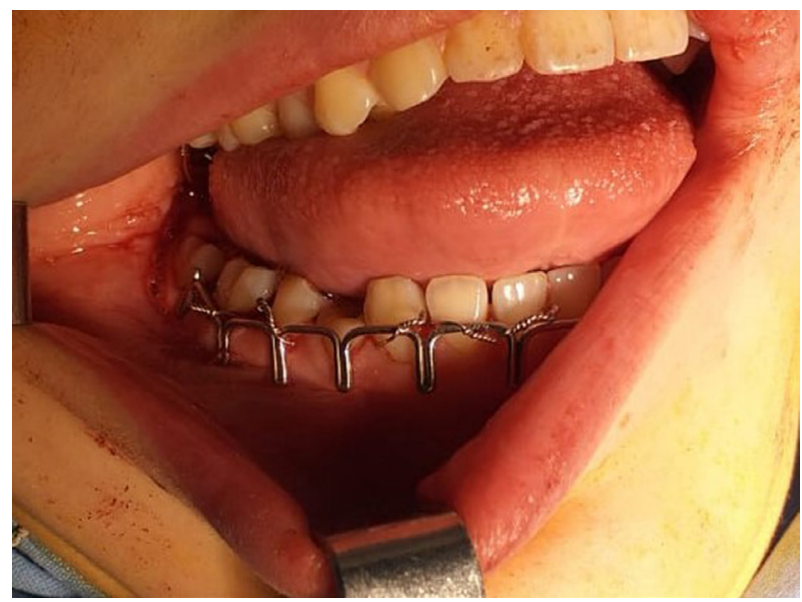

Figura 4. Instalación de arcos peines en el maxilar inferior, para prevención de una fractura patológica intraquirúrgica o posquirúrgica. y escoplo y pinza gubia. Creada la ventana de acceso a la lesión (Figura 6) se procedió a la divulsión roma o legrado de la misma, y resección completa en conjunto con la pieza dentaria 45 , toilette de la cavidad residual (Figura 7) con solución fisiológica estéril atemperada, regularización de márgenes óseos, reposición del colgajo y sutura continua con vycril 4/0 (Figura 8).

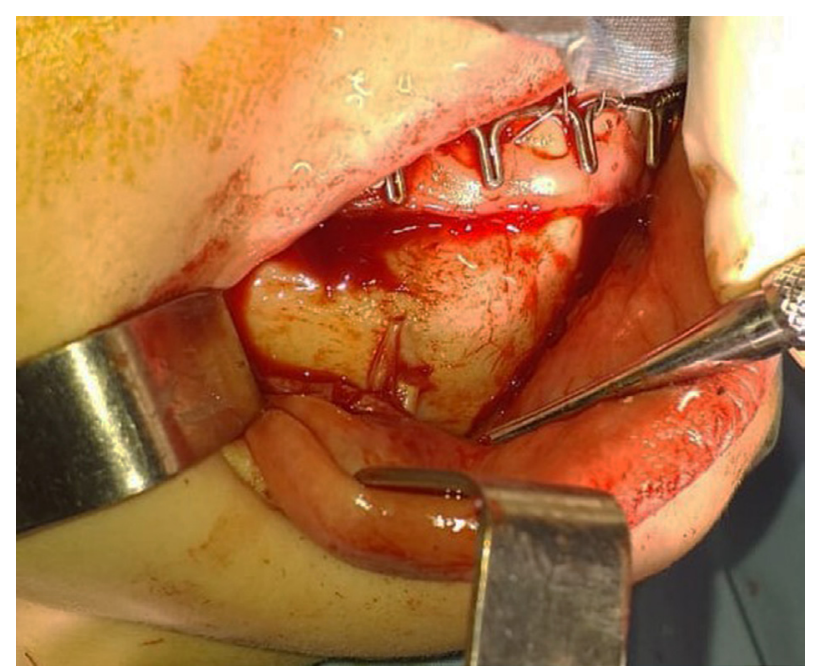

Figura 5. Incisión, legrado y exposición de la salida del paquete Mentoniano. Se puede visualizar que el mismo presenta una bifurcación a la salida por dos orificios.

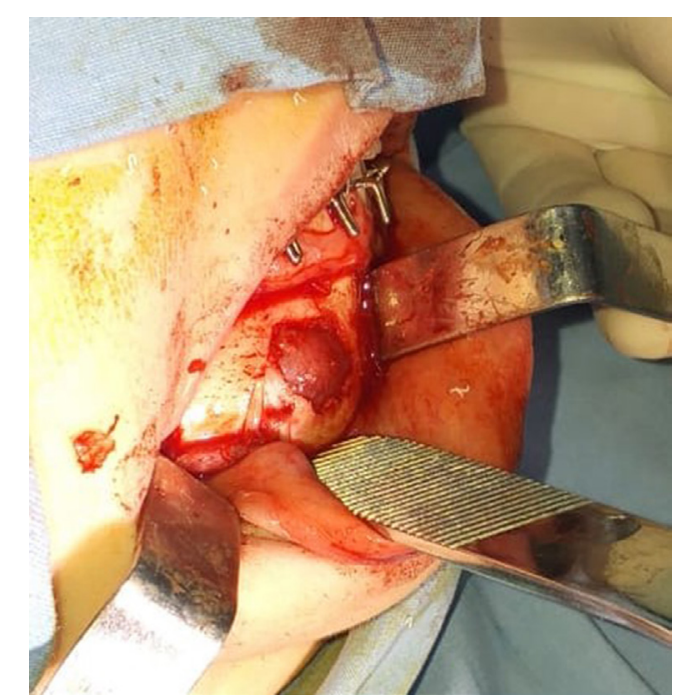

Figura 6. Ostectomía vestibular, exposición del polo externo de la lesión tumoral. 


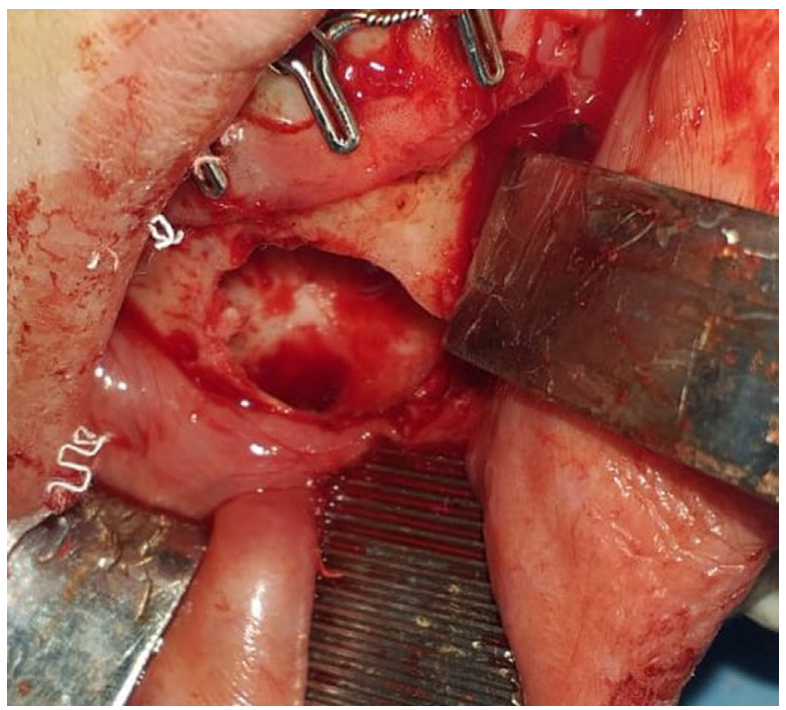

Figura 7. Cavidad extirpación tumoral y de la pieza 44 involucrada. Se conservó el paquete mentoniano intacto.

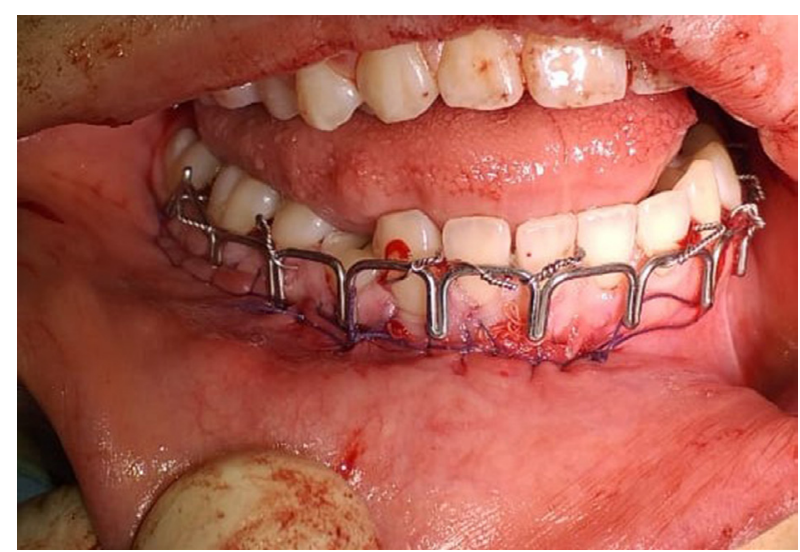

Figura 8. Cierre con sutura vycril 4/0.

Se administró por vía endovenosa analgésico (fentanilo 200 mgr) y antibiótico (cefazolina 2 gr.), continuando por via oral con cefalexina $1 \mathrm{gr}$, y flurbiprofeno 200 AP.

La muestra (Figura 9) se fijó en formol al $10 \%$ y se envió al laboratorio de Anatomía Patológica de la Facultad de Odontología, de la Universidad Nacional de Buenos Aires.

Se indicó el alta de internación a las 12 hs de finalizada la intervención, y se

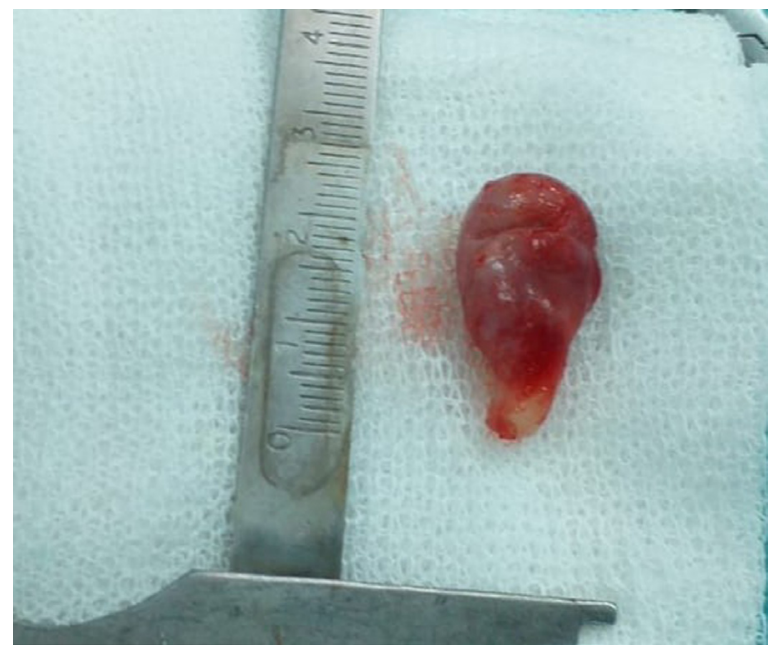

Figura 9. Visualización del Tumor Odontogénico Adenomatoide o TOA de $1,7 \mathrm{~cm}$ de diámetro e inclusión de la pieza 44.

dieron medidas post quirúrgicas en forma verbal y escrita, medicación ambulatoria y programación de controles en el Servicio de Odontología.

En el control postquirúrgico a 48hs la paciente refiriere pérdida de sensibilidad en la zona del mentón.

Se realizó la exploración para demarcar la zona de parestesia mediante la prueba de "pinprick" con la parte aguda de una sonda sobre la zona alterada, ${ }^{16}$ determinando la lesión del nervio dentario inferior en su ramo terminal mentoniano. Se demarcó la parestesia sobre la piel del paciente y se tomaron registros fotográficos para monitorizar con controles clínicos la pérdida o recuperación de la sensibilidad. Se evaluó bajo pruebas de vitalidad dental el ramo incisivo del mismo nervio, resultando positivas, descartando así una posible lesión (Figura 10).

Se evaluó la paciente siguiendo el mismo método, a los 15 y 30 días con evolución favorable y recuperación total de la 


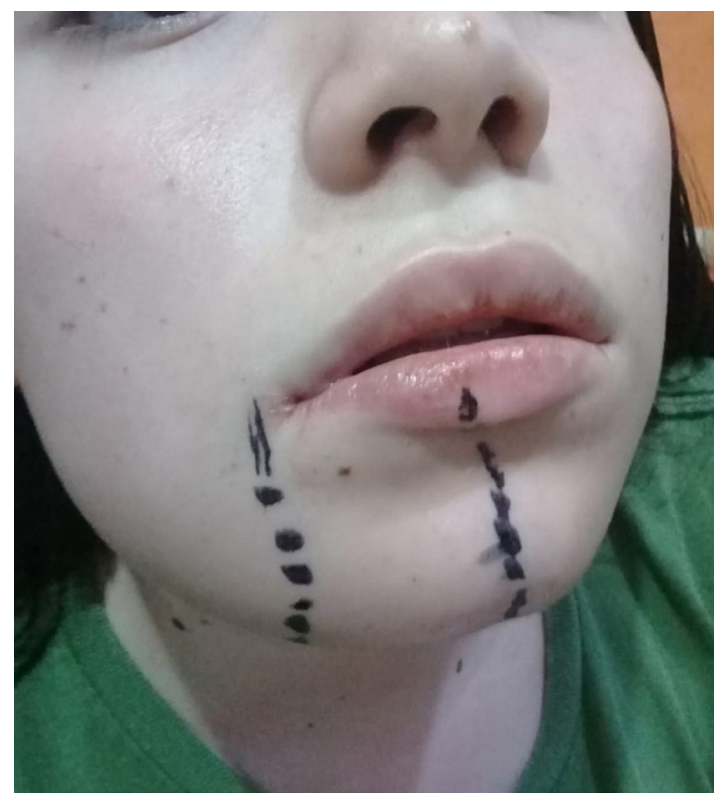

Figura 10. Exploración para demarcar la zona de parestesia mediante la prueba de "pinprick".

sensibilidad del ramo mentoniano en una evaluación 45 días posteriores a la cirugía.

En el examen anatomopatológico macroscópico se identifica, pieza de quistéctomia cerrada de $1,7 \mathrm{~cm}$ de diámetro, asociada a pieza dentaria 44 con inclusión de su corona en la luz de la cavidad quística y unida a la pieza a nivel del límite amelocementario; de superficie externa lisa y blanquecina con áreas ocres. Al corte de consistencia elástica, la luz de la cavidad presenta material grumoso, y en sectores se identifican proyecciones papilares de color pardusco. El espesor de la pared alcanza 0,2 cm. El cuadro histopatológico, en conjunto con las imágenes evaluadas, conlleva al diagnóstico de "tumor odontogénico adenomatoide con áreas pindborgoides" (Figura 11).

Se hizo un seguimiento de control a distancia hasta 10 meses, en donde se observa radiológicamente la remodelación

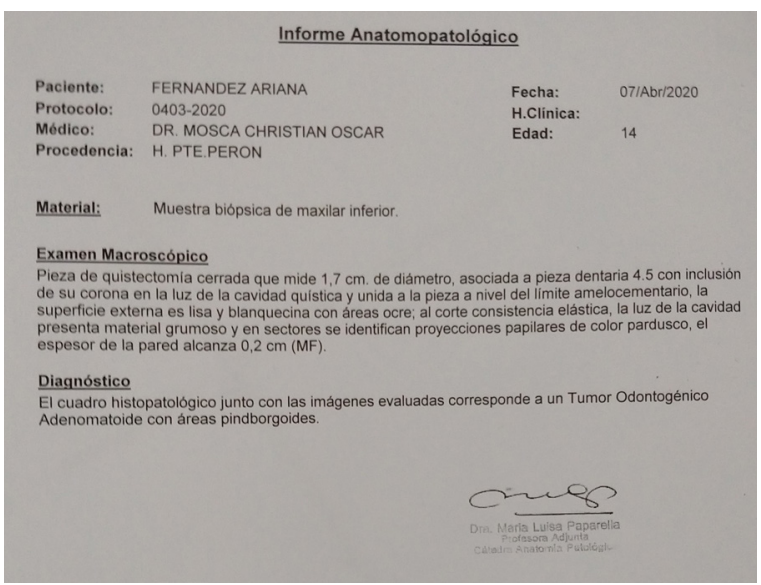

Figura 11. Estudio de Anatomía Patológica con el resultado de TOA o Tumor Odontogénico Adenomatoide.

ósea en la zona donde se encontraba la entidad patológica, sin indicios de recidiva (Figura 12 a y b).
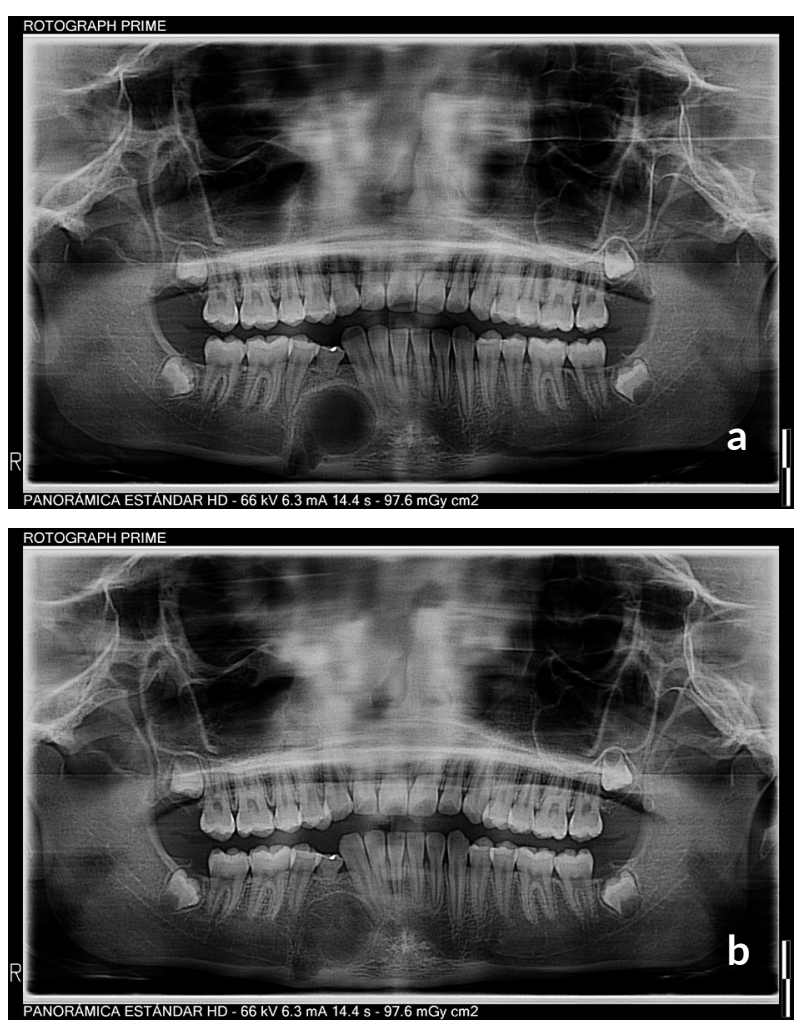

Figura 12. Control por ortopantomografía. a. control inmediato; $b$. control a los 10 meses, en la que se observa la regeneración/remodelado óseo y la ausencia de recidiva 


\section{Discusión}

La OMS en su clasificación de tumores odontogénicos del año 2017, clasificó al TOA como un tumor odontogénico epitelial benigno, ${ }^{7,8}$ poco frecuente, que se presenta principalmente en paciente jóvenes en la segunda y tercera década de vida; evidenciándose una mayor frecuencia en las mujeres, respecto a los varones, siendo la zona con mayor predilección el maxilar superior anterior y suele estar asociado a un diente retenido, generalmente un canino. Uno de los aspectos de interés del caso clínico presentado es el hallazgo de esta entidad en el sector inferior, en premolares, siendo esta presentación poco frecuente. ${ }^{1,17}$

Clínicamente, la característica intraósea o folicular se presenta como una lesión tumoral asintomática, de consistencia dura, bien circunscripta, ${ }^{5}$ crecimiento lento, no agresiva, que rara vez sobrepasa $3 \mathrm{~cm}$ en su dimensión mayor, causando expansión de las corticales óseas, y ocasionalmente asimetría facial y desplazamiento de las raíces de las piezas contiguas, sin invadir los tejidos blandos. ${ }^{15}$ Sin embargo, hay reportes en la bibliografía de casos donde cursa en forma agresiva, adquiriendo gran tamaño, causando lisis de las tablas óseas e incluso rizólisis. ${ }^{18}$

En una amplia revisión realizada por Philipsen, ${ }^{19}$ basándose en 499 casos describe tres variantes de TOA, concluyendo que el tipo folicular intraoseo es el más común y que representa el $70 \%$ de los casos, coincidiendo con el caso expuesto. En la evaluación radiográfica muestra una imagen radiolúcida, unilocular, bien delineada, asociada a la corona de un diente retenido; puede contener múltiples calcificaciones de forma y tamaño variables dispersos o agrupados, o un foco radiopaco, de aspecto redondeado pequeño. La modalidad extraosea folicular se presenta como una radiolucidez unilocular bien definida que se encuentra entre, arriba o superpuesta a las raíces de los diente permanentes erupcionados; ${ }^{15,19}$ la variante extraosea es raramente detectable radiográficamente, pero puede presentarse una ligera erosión de la cresta subyacente del hueso alveolar; ${ }^{15,20}$ observándose como un fibroma o épulis de los tejidos gingivales, con preferencia por el sector mandibular antero inferior. ${ }^{9}$

Histológicamente su patrón más característico se presenta en forma nodular solida de células cuboidales y/o fusiformes de epitelio odontogénico, formando nidos celulares en hojas o estructuras similares a rosetas; esta estructura se encuentra rodeada por un estroma de tejido conectivo bien desarrollado con vasos de paredes delgadas, con presencia de una capa epitelial delgada. En algunas zonas se puede observar material amorfo eosinófilo (calcificaciones distroficas) entre las células epiteliales y en el centro de la estructura en forma de roseta. El tumor puede presentarse como una masa sólida, o parcialmente quístico y generalmente tiene masas globulares de tejido calcificado. ${ }^{15,21,22}$

Hay espacios similares a conductos (pseudoconductos) formados por una fila de células epiteliales cilíndricas con el núcleo polarizado en sentido opuesto al lumen. Dicho lumen puede estar vacío o contener material hialino eosinófilo amorfo ("gotas eosinofilas") o detritos celulares. Las células cuboidales o cilíndricas pueden formar cordones en complicados patrones que puede exhibir invaginación. ${ }^{15,23,24}$ 
De matos, ${ }^{25}$ en el año 2012, reporto que, junto con el TOA, pueden encontrarse áreas de tumor odontogénico epitelial calcificante, caracterizado por calcificaciones esféricas que pueden producir radiográficamente cierto grado de opacidad al tumor, así como también grupos celulares que recuerdan al tumor odontogénico epitelial calcificante (tumor de pindborg), en los que se puede llegar a formar material extracelular eosinófilo, de aspecto amiloideo, ${ }^{25}$ coincidiendo en este aspecto al contrastar con el resultado anatomopatológico obtenido de la biopsia realizada.

Su diagnóstico depende de un hallazgo radiográfico de rutina o por consulta frente a situaciones en la cuales el crecimiento del tumor provoco deformidad facial. ${ }^{26}$

En el diagnóstico diferencial se incluye al quiste dentígero, ameloblastoma uniquístico y tumor odontogénico epitelial calcificante. Las características radiográficas son de una imagen radiolúcida rodeando la corona de una pieza dentaria no erupcionada, de bordes definidos y corticalizados. Pueden presentarse áreas opacas (calcificaciones) ${ }^{27}$ dando aspecto de un tumor odontogénico epitelial calcificante; el diagnóstico diferencial de quiste dentígero se excluye en los casos de TOA en los que la radiolucidez se extiende más allá de la unión corona-raíz. ${ }^{28}$

El tratamiento de elección, es el manejo quirúrgico conservador del tumor, realizando la enucleación en conjunto con la pieza asociada y curetaje simple del hueso circundante, ${ }^{9}$ siendo esta la técnica predilecta por la mayoría de los cirujanos. ${ }^{18} \mathrm{Al}$ ser un tumor benigno, no invasivo localmente, bien encapsulado y presentar baja recurrencia, el pronóstico es excelente. ${ }^{15}$ Algunos sugieren realizar, en determinadas situaciones donde la lesión adquiere grandes dimensiones, una regeneración ósea guiada para evitar asimetrías o atrofias, ${ }^{18}$ si bien disentimos en esto, teniendo en cuenta la edad favorable del paciente para la reparación ósea de la lesión.

Algunos autores indican la técnica descompresiva o marzupialización como tratamiento inicial en situaciones donde la pieza retenida asociada al TOA se encuentra en posición favorable para ser traccionada ortodóncicamente y reposicionada en la arcada dental. Sin embargo, en caso de que el tumor continúe su crecimiento, se indica la remoción inmediata del mismo en conjunto con el órgano dental. ${ }^{9}$

\section{Conclusión}

El pronóstico del TOA es bueno, la tasa de recurrencia es de $0,2 \%$ Solo se reportan cuatro casos de recurrencia en la literatura. ${ }^{9,29}$

En nuestro caso la decisión fue de una cirugía conservadora sin regeneración ósea debido a la edad de la paciente, y la cercanía del paquete vasculonervioso dentario inferior y su ramal terminal mentoniana, en donde los controles posquirúrgicos demostraron una buena evolución con recuperación de la sensibilidad y la no recidiva de la entidad tumoral.

\section{Declaración}

Declaramos que para el presente trabajo no existe conflicto de interés, ni tampoco hubo financiación privada o pública. 


\section{Referencias bibliográficas}

1. Acuña-Pérez D, Bodaño-Gómez NN. Tumor odontogénico adenomatoide en región mandibular. Revista Electrónica Dr. Zoilo E. Marinello Vidaurreta. 2019 Julio-Agosto; Vol. 44(Número 4). http://revzoilomarinello.sld. cu/index.php/zmv/article/view/1820

2. Rey E. Cirugía Bucomaxilofacial. Primera Edición ed. Ciudad Autonoma de Buenos Aires: Corpus Libros Médicos y Científicos; 2020.

3. Harbitz F. On cystic tumors of the maxilla, and especially on adamantine cystadenomas (adamantomas). Dent Cosmos 1915;57:1081-93.

4. Castillejos RD, Munguía A, Ham GC. Tumor odontogénico adenomatoide. Reporte de un caso y revisión de la literatura. Revista Odontológica Mexicana. 2015 Julio-Septiembre; 19: 3.

5. Sempere FJV, Martinez MJA, Sirera BV, Marco JB. Tumor odontogenico adenomatoide folicular: estudio inmunohistoquimico. Medicina y Patología Oral. 2006 Mar; 11: E305-8.

6. Vila CN. Cirugia oral y maxilofacial. In Vila CN. Cirugia oral y maxilofacial. Segunda ed. Madrid: ARAN ediciones S.L; 2009. p. 33-52.

7. Wright JM, Vered M. Update from the 4th Edition of the World Health Organization. Head and Neck Pathol. 2017 February; 11:68-77. DOI 10.1007/s12105-017-0794-1

8. Wright JM, Tekkesin MS. Odontogenic tumors: where are we in 2017. Journal of Istanbul University Faculty of Dentistry. 2017 Diciembre 2;: p. 51 (3 Supl. 1): S10 - S30. DOI: 10.17096/jiufd.52886

9. Dezza Palle J, Arce Lazo M. Tumor odontogénico: repote de caso. Odontologia Sanmarquina. 2018 Marzo 21; 21(1): 41-46. https://www.researchgate.net/publication/326027499_Tumor_odontogenico_adenomatoide_ Reporte_de_caso

10. Alatorre PS. Tumor odontogénico adenomatoide. Reporte de un caso. Rev Mex Cir Bucal Maxilofac. 2016 EneroAbril; 12(1): 21-27. https://www.medigraphic.com/cgi-bin/new/resumen.cgi

11. Vélez J. Tumor odontogénico adenomatoide extrafolicular de localización mandibular. Presentacion de caso. Revista Cubana de Estomatología. 2018 Mayo; 55 (3).

12. Sneha Sethi. A case report and short review on changing trends in the site of occurrence of adenomatoid odontogenic tumor: Unravelling the past 15 years. Dent Res J (Isfahan). 2016 Septiembre; 13(5): 462-471.

13. Velasco I. Tumor Odontogénico Adenomatoide en Maxilar: Reporte de un Caso y Revisión de la Literatura. Int. J. Odontostomat. 2011 Abril; 5(1): 65-69.

14. Queija Saldaña MA, Vinicius de Oliveira M, Barbosa Martins V, Cavalcanti de Albuquerque G, Motta Júnior J. Tumor odontogénico adenomatoide extrafolicular: reporte de caso. Revista Cientifica Odontología Sanmarquina. 2020; 23(1): 51-55.

15. Marín Botero ML. Tumor odontogénico adenomatoide. Reporte de un caso y revisión de la literatura. Av. Odontoestomatol. 2017 Enero 10; 33 (4): 161-170.

16. Cosme Gay Escoda. Cordales incluidos. Exodoncia quirúrgica. Complicaciones. In Ergon, editor. Tratado de cirugía bucal. 2011th ed. Madrid: Ediciones Ergón, S.A.; 2004. 387-457.

17. Oriana Valenzuela Rivera. Tumor Odontogénico Adenomatoide: Reporte de dos casos de localización mandibular. Revista de Odontopediatria Latinoamericana. 2013; 3 (1).

18. Manuel Escalante Fontalvo. Concepto actual, diagnóstico y tratamiento del tumor odontogénico adenomatoide. Reporte de un caso. Revista Científica Salud Uninorte. 2012 septiembre-diciembre; 28 (3).

19. Philipsen HP. Adenomatoid odontogenic tumor: biologic. J Oral Pathol Med. 1991; 20(4): 149-58.

20. Rick GM. Adenomatoid odontogenic tumor. Oral Maxillofac Surg Clin North Am. 2004 Aug;16(3): 333-54. doi: 10.1016/j.coms.2004.04.001. PMID: 18088735.

21. Komal K VA. Mural adenomatoid odontogenic tumor in the mandible: A rare case. Int J Oral Maxillofac Pathol. 2011; (2): 35-9..

22. Yilmaz N. Extrafollicular adenomatoid odontogenic. Eur J Dent. 2009; 3 (1): 71-4.

23. Jindwani K. Surgical management of peripheral variant of adenomatoid odontogenic tumor: A rare case report with review. Contemp Clin Dent. 2015; 6(1): 128-30.

24. Philipsen HP. Adenomatoid odontogenic tumour. In Leon Barnes J, editor. World Health Organization Classification of Tumours: Head and Neck Tumours. Lyon; 2005. 304-5.

25. de Matos FR. Adenomatoid odontogenic tumor: retrospective study of 15 cases with emphasis on histopathologic features. HeadNeck Pathol. 2012; 6(4): 430-7.

26. Duque Serna Francisco Levi, Castillón Pino Liliana, Ardila Medina Carlos Martin. Caracterización del tumor odontogénico adenomatoide en una población Colombiana. AMC [Internet]. 2013; 17( 2): 187-200. Disponible en: http://scielo.sld.cu/scielo.php?script=sci_arttext\&pid=S1025-02552013000200010\&lng=es.

27. Rosa AC. A combined epithelial odontogenic tumor? A 7-year follow-up case. Head Neck Pathol. 2017; 11(4): 519-24. 
28. Becker T. Critical evaluation of the radiological and clinical features of adenomatoid odontogenic tumor. Dentomaxillofac Radiol. 2012; 41: 533-540.

29. Zhou Chuan-Xiang GY. Adenomatoid odontogenic tumor: a report of a rare case. J Oral Pathol Med. 2007; 36: 440-3.

Recibido: $18 / 02 / 21$

Aceptado: $19 / 07 / 21$

Correspondencia: Dr. Christian O. Mosca, correo: moscach@yahoo.com 